\title{
A REMARK ON A THEOREM OF MARSHALL HALL
}

\author{
M. F. SMILEY
}

Marshall Hall [2] ${ }^{1}$ proved that the identity

$$
x(y z-z y)^{2}=(y z-z y)^{2} x
$$

characterizes quaternion algebras among associative but not commutative division rings. Our remark is that (1) characterizes CayleyDickson algebras among alternative but not associative division rings. This follows from Hall's proof and a result of A. A. Albert [1]. A theorem of R. D. Schafer [4, Theorem 4] permits us to conclude that (1) and Hall's Theorem L [2] (as universal) ensure that the coordinate ring of a projective plane is uniquely defined irrespective of the coordinate system.

It is easy to verify (1) in a Cayley-Dickson algebra. We should like to sketch a proof of the converse, which is independent of Albert's result in [1]. Let $A$ be an alternative division ring and let $F$ be the set of all elements $c \in A$ which satisfy $c x=x c$ for every $x \in A$. Then $F$ is a field ${ }^{2}$ and, when 3 is nonzero in $A, F$ is the center of $A[3 ; 5$, Lemma 9]. If $A$ satisfies (1), the proof of Hall's Lemma 1 [2, p. 262] yields $x^{2}=t(x) x-n(x)$ for every $x \in A$ not in $F$, where $t(x), n(x) \in F$. Define $t(c)=2 c, n(c)=c^{2}$ for $c \in F$.

When 3 is zero in $A$ and $A$ satisfies (1), $F$ is still the center of $A$ as we shall now show. ${ }^{3}$ Since 2 is a nonzero in $A$, for each $x \in A$ we have $(x+c)^{2} \in F$ for some $c \in F$. If, then, $c_{1}, c_{2}$ are in $F$, we have $\left(c_{1}, c_{2}\right.$, $\left.(x+c)^{2}\right)=0$. By use of some identities of Zorn [6, (1.6) and (1.8); 5 , (1)] we find that $\left(c_{1}, c_{2},(x+c)^{2}\right)=2(x+c)\left(c_{1}, c_{2}, x+c\right)=2(x+c)$ $\cdot\left(c_{1}, c_{2}, x\right)$. We infer that $\left(c_{1}, c_{2}, x\right)=0$ for every $c_{1}, c_{2}$ in $F$ and every $x$ in $A$. Now let $c \in F$ and $x, y \in A$. As before, $\left(x+c^{\prime}\right)^{2} \in F$ for some $c^{\prime} \in F$ and

$$
0=\left(c,\left(x+c^{\prime}\right)^{2}, y\right)=2\left(x+c^{\prime}\right)\left(c, x+c^{\prime}, y\right)=2\left(x+c^{\prime}\right)(c, x, y),
$$

so that $(c, x, y)=0$ as desired.

To complete the argument, we first prove (i) $t(x+y)=t(x)+t(y)$,

Presented to the Society, February 26, 1949; received by the editors January 11, 1949 and, in revised form, February 14, 1949.

1 Numbers in brackets refer to the references cited at the end of the paper.

2 To verify that $c, c^{\prime} \in F$ yields $c c^{\prime} \in F$ even if $3=0$ in $F$, we may use the equations $\left(x, c, c^{\prime}\right)=\left(c^{\prime}, x, c\right)=\left(c, c^{\prime}, x\right)$, where $(x, y, z)=x(y z)-(x y) z$ is the associator of $x, y$, and $z$.

We are indebted to the referee for his observation that our original proof was incomplete in this case and also for simplifying our amended version. 
(ii) $t(c x)=c t(x)$, and (iii) $n(x y)=n(x) n(y)$ for every $x, y \in A$ and every $c \in F$, and then we apply the arguments of R. D. Schafer [4]. We indicate the proof of (i) and (iii) when $x y \neq y x$. We compute $(x+y)^{2}=x^{2}+x y+y x+y^{2}=t(x+y)(x+y)-n(x+y)$, and we obtain (i) by taking the commutator with $x$ and then (iii) by multiplying by $x y$ on the left.

Addendum (October 25, 1949). When $1+1=0$ in $A$, we cannot obtain our result from Albert's Theorem 1 of [7] which employs $1+1 \neq 0$. In this case we must complete the proof of (i)-(iii) by using Lemma 2 of $[2$, p. 262] and some straightforward but rather lengthy calculations. In fact, we may regard our proof as establishing Albert's Theorem 1 even when $1+1=0$. First redefine quadratic algebra by deleting the coefficient 2 in $f(\xi, x)$. Then assume that $A$ is quadratic and alternative and observe that $A$ is a division algebra. Unless $A$ is commutative and hence a field over $F$, one may use the arguments of Hall [2, pp. 262-263] to see that the center of $A$ is $F$. For, if $x y \neq y x$, then $Q=F[1, x, y, x y]$ is a quaternion algebra over $F$, and the requirement that $c \in A$ commute and associate with the elements of $Q$ implies that $c \in Q$ and hence that $c \in F$, since the center of $Q$ is $F$. We are now in a position to apply the arguments of the present note.

\section{REFERENCES}

1. A. A. Albert, Absolute valued algebraic rings, Bull. Amer. Math. Soc. vol. 54 (1948) p. 1050.

2. Marshall Hall, Projective planes, Trans. Amer. Math. Soc. vol. 54 (1943) pp. 229-277.

3. N. Jacobson, Structure theory of simple rings without finiteness assumptions, Trans. Amer. Math. Soc. vol. 57 (1945) pp. 228-245.

4. R. D. Schafer, Alternative algebras over an arbitrary field, Bull. Amer. Math. Soc. vol. 49 (1943) pp. 549-555.

5. M. F. Smiley, The radical of an alternative ring, Ann. of Math. (2) vol. 49 (1948) pp. 702-709.

6. Max Zorn, Alternative rings and related questions I: Existence of the radical, Ann. of Math. (2) vol. 42 (1941) pp. 676-686.

7. A. A. Albert, Absolute-valued algebraic algebras, Bull. Amer. Math. Soc. vol. 55 (1949) pp. 763-768.

State University of Iowa 